\title{
Apoptosis in the chorion laeve of term patients with histologic chorioamnionitis
}

\author{
A. P. Murtha ${ }^{1}$, R. Auten ${ }^{2}$ and W. N. P. Herbert ${ }^{1}$ \\ ${ }^{1}$ Division of Maternal Fetal Medicine, Department of Obstetrics and Gynecology, and \\ ${ }^{2}$ Division of Neonatal Medicine, Department of Pediatrics, Duke University Medical Center, Durham, NC
}

\begin{abstract}
Objective: The balance between cell survival and cell death (apoptosis) is critical during development and may affect organ function. Apoptosis is accelerated in the presence of infection and inflammation in a variety of organ systems. The objective of this investigation was to determine if apoptosis was increased in the chorion laeve of term patients with and without histologic chorioamnionitis.

Methods: Records of placental pathology were reviewed with respect to the presence/absence of histologic chorioamnionitis. Sections from formalin-fixed, paraffin-embedded fetal membrane rolls were stained using the TUNEL method. The proportion of apoptotic nuclei was calculated in seven high-powered fields/section. Those with and without histologic chorioamnionitis were compared. Data were analyzed using the Mann-Whitney $U$ test, with significance defined as $p<0.05$.

Results: There was no significant difference in demographic or clinical characteristics between the two groups. The chorion laeve from subjects with histologic chorioamnionitis had significantly more apoptotic nuclei when compared to those without chorioamnionitis (11.2\% vs. $5 \%, p=0.02$ ).

Conclusion: Apoptosis is more prevalent in the chorion laeve of fetal membranes with histologic chorioamnionitis. This finding suggests that infection/inflammation may impact cell survival within fetal membranes. The implications of these findings warrant further investigation.
\end{abstract}

Key words: Apoptosis; Fetal Membranes; ChORIOn LAeve; CHORIOAmNiOnitis

The balance between cell survival and death is critical to the maintenance of normal tissue. Apoptosis or physiologic cell death plays a critical role in development and tissue homeostasis ${ }^{1}$. Apoptosis is an active, energy-requiring process that occurs in certain cells and is regulated by a complex molecular signaling mechanism. In pathologic conditions, these same molecular signaling pathways may be induced resulting in acceleration of cell death. Examples of induction of apoptosis in pathologic states include the response of the central nervous system to oxidative stress and the response of the cardiovascular system to ischemia and hypoxia ${ }^{1}$. In neutrophils, there is a unique balance between cell death and cell survival regulated by inflammatory mediators that serve to protect the host. For example, substances such as lipopolysaccharide (LPS) and cytokines such as granulocyte colony stimulating factor (G-CSF) and interleukin-8 (IL-8) receptor agonists have been shown to inhibit neutrophil apoptosis ${ }^{2}$. In addition, tumor necrosis factor- $\alpha$ (TNF- $\alpha$ ) and nitric oxide donors induce apoptosis that results in loss of the neutrophil before degranulation therefore limiting the inflammatory response ${ }^{3}$. These mechanisms serve to protect the host against both 
microbial invasion and excessive prolongation of inflammation.

Apoptosis appears to be accelerated by inflammation and infection. In vivo studies suggest that in sepsis models, parenchymal tissue undergoes accelerated apoptosis. In response to intravenous (I.V.) injection of LPS, Bohlinger and colleagues ${ }^{4}$ noted apoptosis in the liver, lung, kidney and intestine of mice with the extent of apoptosis correlating with serum TNF- $\alpha$ levels. Additionally, in septic human patients, Hotchkiss and co-workers ${ }^{5}$ noted focal apoptosis in the spleen, colon and ileum; and a significant increase in cleaved 'caspase', an important enzyme in the induction of apoptosis, in the spleens of septic compared to non-septic patients. Infection and inflammation may alter the delicate balance between cell death and cell survival resulting in diminished function of the organ system.

The study of apoptosis in pregnancy has focused on the placenta and fetal membranes. In a retrospective histologic examination of fetal membranes, Parmley ${ }^{6}$ reported widespread cell death and loss of the chorion laeve as term approached. By 36 weeks, there were foci where chorion cells were absent and the decidua was in direct contact with the fetal connective tissues. In the presence of chorioamnionitis, this same tissue layer was prematurely destroyed. The mechanisms involved in the destruction of this cell layer and the impact on the structure and function are not understood.

Fetal membranes, which play an integral role in the maintenance of pregnancy, are composed of two fetal layers. The amnion is a single epithelial cell layer, which provides the tensile strength. The chorion laeve is a metabolically active layer that produces enzymes that inhibit uterine contractions by degrading such substances as prostaglandins, oxytocin, endothelin and platelet activating factor ${ }^{7}$.

With these considerations, we hypothesized that apoptosis in the chorion laeve of fetal membranes may be increased in the presence of chorioamnionitis thereby resulting in an imbalance between cell death and survival. Ultimately, loss of the protective effect of fetal membranes may occur. Specifically we sought to determine whether apoptosis occurs in the chorion laeve of fetal membranes at term and, further, to determine if there is increased apoptosis in the presence of histologic chorioamnionitis.

\section{SUBJECTS AND METHODS}

All pathology reports on placental/fetal membrane specimens submitted from January 1-June 30 1997 were reviewed. The presence or absence of histologic chorioamnionitis was observed. Hospital records were reviewed and subjects included if there was absence of both pre-existing medical conditions, including diabetes, hypertension, lupus and HIV, and pregnancy complications, including pre-eclampsia, abruption, gestational diabetes and severe chorioamnionitis. All samples were 37-41 weeks gestation and were paired for presence or absence of histologic chorioamnionitis. Diagnosis of histologic chorioamnionitis was determined using criteria described by Salafia and colleagues ${ }^{8}$, including infiltration of neutrophils in the chorion and amnion of the extra-placental membranes. All membrane rolls were reviewed by the staff pathologist.

Formalin-fixed (10\% phosphate buffered), paraffin-embedded fetal membrane samples from 39 term pregnancies with and without histologic chorioamnionitis were stained using the TUNEL method (terminal deoxynucleotidyl transferasemediated deoxyuridine triphosphate nick endlabeling) (Oncor, NY). This labels the 3'-hydroxyl $(\mathrm{OH})$ groups of deoxyribonucleotides using terminal transferase and digoxigenin-labeled deoxyuridine triphosphate. The $3^{\prime}-\mathrm{OH}$ ends are generated as a result of endonuclease activity characteristic of apoptosis. The end-labeled DNA is then detected in situ with an anti-digoxigenin: peroxidase conjugate antibody and detected with diaminobenzidine (DAB) substrate. This method is widely used as an immunohistologic marker for apoptosis.

Apoptotic nuclei were defined as TUNELstained positive nuclei in the chorion laeve with histologic characteristics consistent with apoptosis, specifically nuclear condensation. Quantification of apoptosis was performed by counting the number of apoptotic nuclei relative to normal nuclei in seven random, high-powered fields (hpf) of the chorion laeve. The investigator was masked to the identity of the samples until all data were 
collected. The percentage of apoptotic nuclei was calculated by dividing the total number of apoptotic nuclei by the total number of nuclei counted in seven hpf per section. Data were analyzed using the Mann-Whitney $U$ test, Fisher exact and $t$-test with significance defined as $p<0.05$.

\section{RESULTS}

There was no difference in maternal age, race, insurance, marital status, gestational age, length of rupture of membranes, artificial versus spontaneous rupture of membranes or Cesarean delivery rate between the two groups (Table 1).

There was no difference in the total number of nuclei counted in each group. Fetal membrane samples with histologic evidence of chorioamnionitis had significantly more apoptotic nuclei than those without histologic chorioamnionitis $(p=0.1)$. In addition, the percentage of apoptotic nuclei was significantly higher in the chorioamnionitis group when compared to the group without chorioamnionitis (11.2 versus 5\%, $p=0.02)$ (Table 2).

\section{DISCUSSION}

Normal tissue function is dependent on the structure and function of the individual cells that compose that tissue. This homeostasis is maintained by a genetically controlled molecular signaling pathway that results in programmed cell death, without producing a destructive inflammatory response. Alterations in the balance between cell death and survival occur when these pathways are modified by various mechanisms. Pathologic imbalance between cell death and survival may hinder the ability of that tissue to function normally.

The results of this investigation suggest that, in the presence of histologic chorioamnionitis, there is a doubling in the extent of apoptosis in the chorion laeve of fetal membranes. This finding suggests that the presence of infection or inflammation may alter the balance between cell death and survival in the fetal membranes. As fetal membranes serve an important role in the maintenance of pregnancy, such alteration in the balance
Table I Demographic and clinical characteristics

\begin{tabular}{lccc}
\hline & \multicolumn{2}{c}{ Chorioamnionitis } & \\
\cline { 2 - 3 } Characteristic & Present & Absent & p-value \\
\hline Age (mean; years) & 24.8 & 23.8 & NS \\
Non-white (\%) & 46 & 54 & NS \\
Medicaid (\%) & 50 & 50 & NS \\
Single (\%) & 50 & 50 & NS \\
Gestational age (mean; weeks) & 39.4 & 38.9 & NS \\
Cesarean delivery (\%) & 48 & 52 & NS \\
SROM (\%) & 47 & 53 & NS \\
Length of ROM (mean; hours) & 9.7 & 7.0 & NS \\
\hline
\end{tabular}

NS, non-significant; SROM, spontaneous rupture of membranes; ROM, rupture of membranes; data analyzed by Fisher exact or t-test

Table 2 Apoptotic nuclei in chorion laeve

\begin{tabular}{|c|c|c|c|}
\hline \multirow[b]{2}{*}{ Nuclei reported } & \multicolumn{2}{|c|}{ Chorioamnionitis } & \multirow[b]{2}{*}{ p-value } \\
\hline & Present & Absent & \\
\hline Total samples & 18 & 21 & \\
\hline $\begin{array}{l}\text { Total nuclei-mean } \\
\quad \text { (range) }\end{array}$ & $\begin{array}{c}997 \\
(682-1403)\end{array}$ & $\begin{array}{c}957 \\
(643-1285)\end{array}$ & NS \\
\hline \multicolumn{4}{|l|}{ Total apoptotic } \\
\hline $\begin{array}{l}\text { nuclei-mean } \\
\text { (range) }\end{array}$ & $\begin{array}{c}89 \\
(10-230)\end{array}$ & $\begin{array}{c}39 \\
(15-121)\end{array}$ & 0.01 \\
\hline \multicolumn{4}{|c|}{ Percentage of apoptotic } \\
\hline $\begin{array}{l}\text { nuclei-mean } \\
\text { (range) }\end{array}$ & $\begin{array}{c}11.2 \% \\
(0.9-36.7)\end{array}$ & $\begin{array}{c}5.0 \% \\
(1.3-15.1)\end{array}$ & 0.02 \\
\hline
\end{tabular}

NS, non-significant; data analyzed by $t$-test or Mann-Whitney $\cup$ test

between cell death and survival may result in diminished function. Specifically, the chorion laeve of fetal membranes serves an important protective function for the maintenance of pregnancy, including the destruction of substances that would typically result in uterine contractions. With infection or inflammation, the balance between cell death and cell survival is disturbed and the protective effect of the chorion laeve may be lost.

The subjects in this investigation all had uncomplicated pregnancies beyond 37 weeks gestation. Therefore, the role of apoptosis played in preterm labor or preterm premature rupture of membranes (pROM) cannot be addressed by this study. Fortunato and co-workers ${ }^{9}$ have reported that apoptosis may play an important role in preterm 
$\mathrm{pROM}$ and that this mechanism is likely distinct from preterm or term labor. In that study they demonstrated an increased expression of bax and $p 53$ (pro-apoptotic) but decreased expression of bcl-2 (anti-apoptotic) in patients with preterm pROM when compared to preterm labor and term labor patients with intact membranes. The authors suggest that accelerated apoptosis leads to destruction of the normal fetal membranes ${ }^{9}$. The role of specific inflammatory mediators and infection in this process remains unclear.

Based on this information it is apparent that cell death is altered in pathologic states during pregnancy. While it is likely that apoptosis plays a role in the pathophysiology of adverse pregnancy outcome the exact role is not known. Additional investigations are needed to improve our understanding of the role of apoptosis and the potential impact of an imbalance between cell survival and cell death on membrane structure and function. There is mounting evidence that chronic inflammation is present in pregnancies that result in preterm delivery. The impact of chronic inflammation on cell survival in the fetal membranes may be critical to the understanding of the pathophysiology of these conditions. With improved understanding of these processes, new and innovative treatment modalities may be discovered.

\section{REFERENCES}

1. Kam PC, Ferch NI. Apoptosis: mechanisms and clinical implications. Anaesthesia 2000;55:1081-93

2. Auten RL, Richardson RM, White JR, et al. Nonpeptide CRCX2 antagonist prevents neutrophil accumulation in hypoxia-exposed newborn rats. J Pharmacol Exp Ther 2001;299:1-6

3. Haslett C. Granulocyte apoptosis and inflammatory disease. Br Med Bull 1997;53:669-83

4. Bohlinger I, Leist M, Gantner F, et al. DNA fragmentation in mouse organs during endotoxic shock. Am J Pathol 1996;149:1381-93

5. Hotchkiss RS, Swanson PE, Freeman BD, et al. Apoptotic cell death in patients with sepsis, shock and multiple organ dysfunction. Crit Care Med 1999;27:1230-51

ReCeIVED 09/14/01; ACCEPTED 12/28/01
6. Parmley TH. Spontaneous cell death in the chorion laeve. Am J Obstet Gynecol 1990;162:1576-83

7. Germain AM, Smith J, Casey ML, et al. Human fetal membrane contribution to the prevention of parturition: uterotonin degradation. J Clin Endocrinol Metab 1994;78:463-70

8. Salafia CM, Weigl C, Silberman L. The prevalence and distribution of acute placental inflammation in uncomplicated term pregnancies. Obstet Gynecol 1989;73:383-9

9. Fortunato SJ, Menon R, Bryant C, et al. Programmed cell death (apoptosis) as a possible pathway to metalloproteinase activation and fetal membrane degradation in premature rupture of membranes. $\mathrm{Am}$ J Obstet Gynecol 2000;182:1468-76 


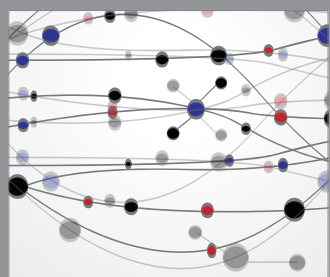

The Scientific World Journal
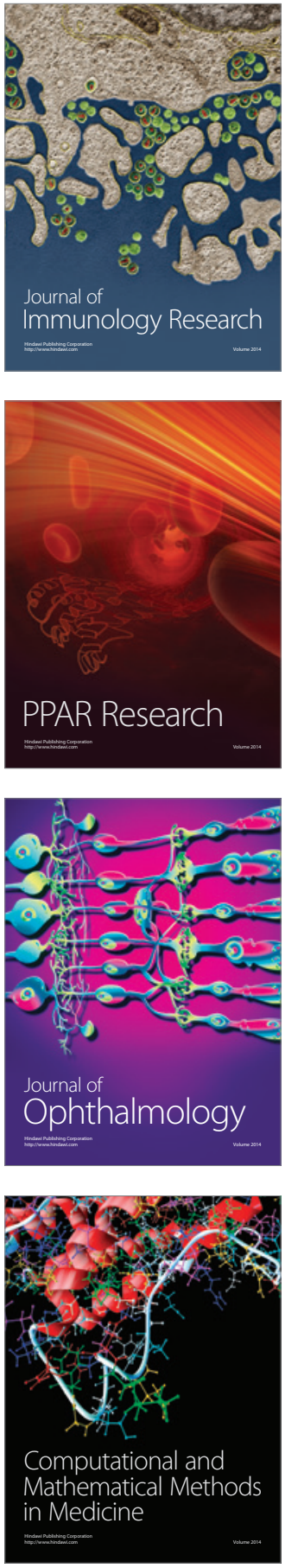

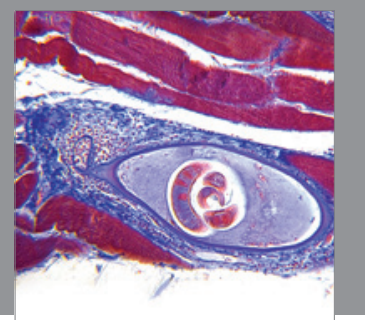

Gastroenterology

Research and Practice
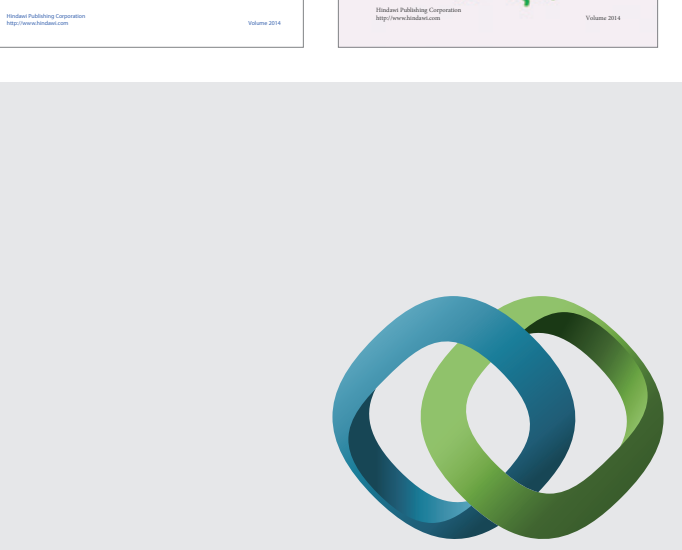

\section{Hindawi}

Submit your manuscripts at

http://www.hindawi.com
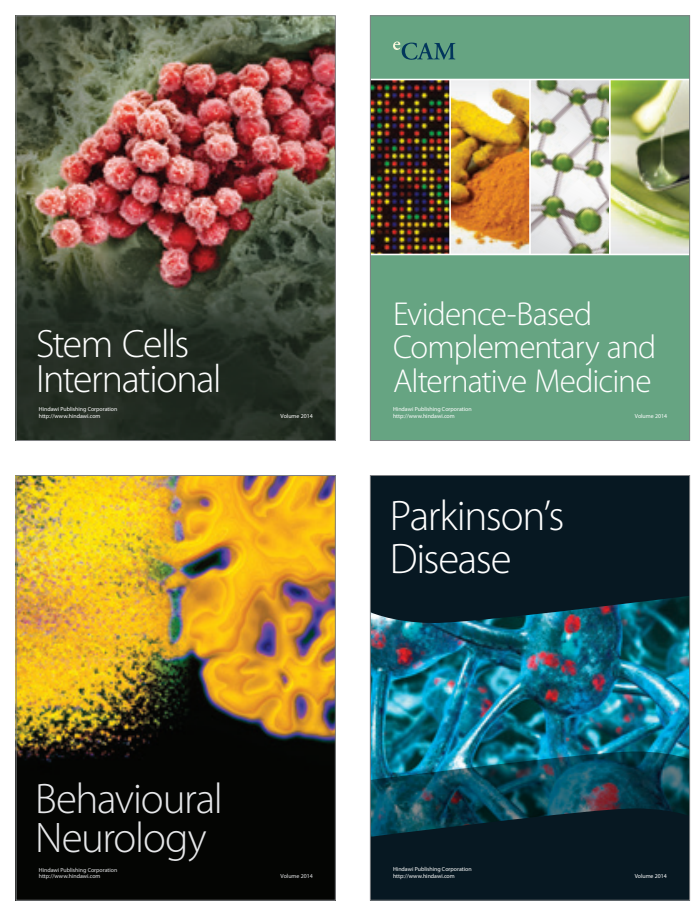

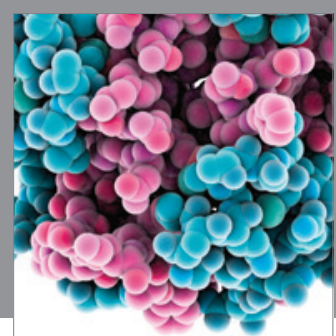

Journal of
Diabetes Research

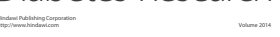

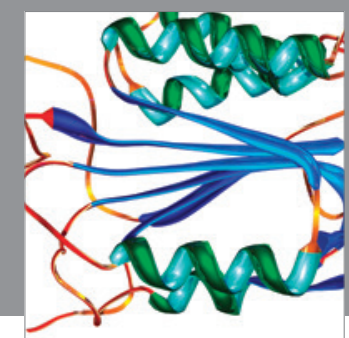

Disease Markers
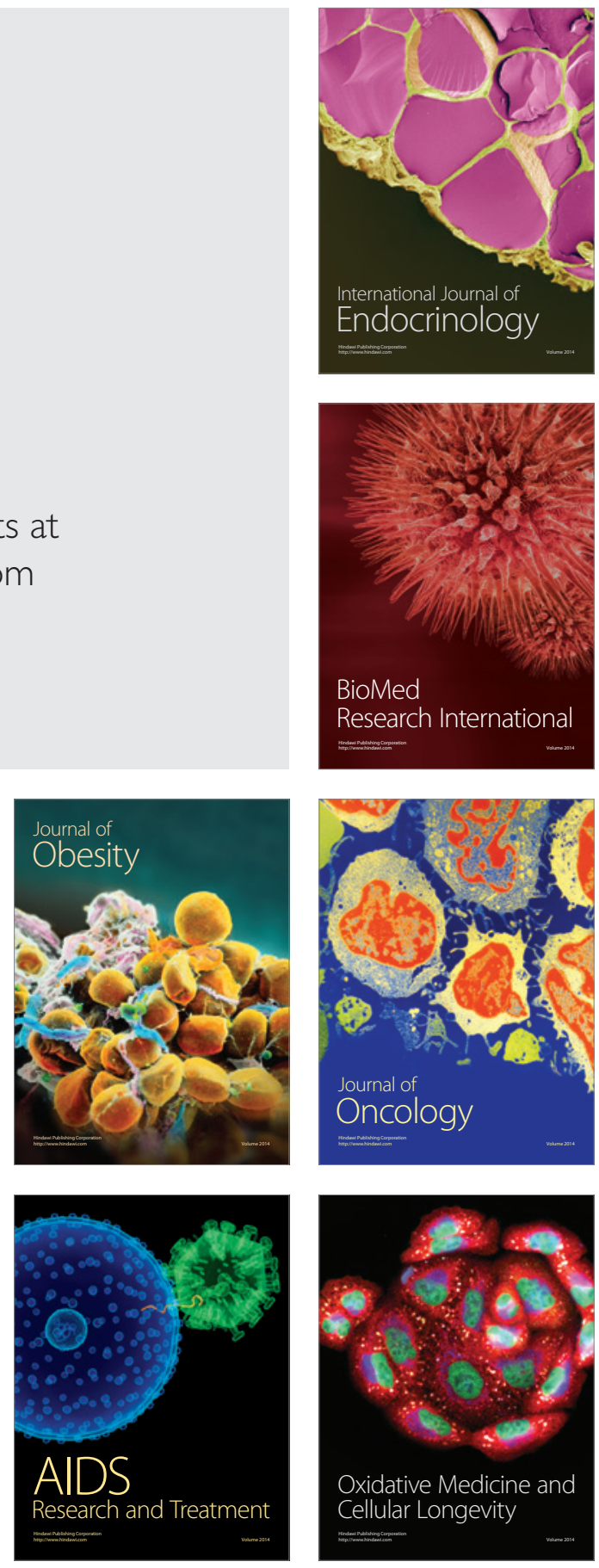